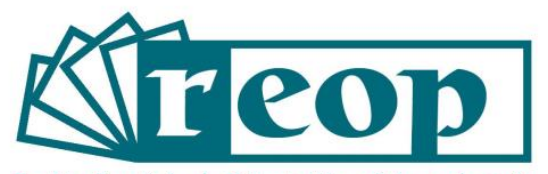

Revista Española de Orientación y Psicopedagogía

\title{
DISEÑO Y APLICACIÓN DE ESTRATEGIAS NEUROPSICOPEDAGÓGICAS EN ESTUDIANTES UNIVERSITARIOS
}

\section{DESIGN AND APPLICATION OF NEUROPSYCHOPEDAGOGICAL STRATEGIES IN UNIVERSITY STUDENTS}

\author{
María de los Ángeles Sánchez Trujillo ${ }^{1}$ \\ Universidad Peruana de Ciencias Aplicadas. Lima, Perú \\ Eduar Antonio Rodríguez Flores \\ Universidad Tecnológica del Perú. Lima, Perú
}

\section{RESUMEN}

Esta investigación responde a la necesidad de atender aquellas falencias cognitivas y socioemocionales que puedan estar dificultando el proceso de adaptación de estudiantes recién ingresantes a la universidad. A partir de lo anterior, el objetivo general de este estudio fue describir los logros y limitaciones de una propuesta de estrategias neuropsicopedagógicas diseñadas a partir de un diagnóstico inicial $y$, posteriormente, aplicadas a estudiantes universitarios. En tal sentido, el presente trabajo responde a un enfoque cualitativo y corresponde a una investigación-acción, la que permitió resolver algunas problemáticas detectadas en el caso de estudio, el cual estuvo conformado por 43 estudiantes de primer ciclo de la Facultad de Educación de una universidad privada de Lima. A fin de detectar sus falencias, se emplearon

\footnotetext{
1 Correspondencia: María de los Angeles Sánchez Trujillo. Dirección postal completa: Avenida Felipe Salaverry 575, departamento 1904. Jesús María, Lima, Perú. Teléfono: 945682957. Correo-e: pchemsan@upc.edu.pe
} 
guías de entrevistas semi-estructuradas a sus docentes, así como una encuesta de opinión dirigida a los estudiantes. Ello sirvió de insumo para el diseño de estrategias, las cuales fueron evaluadas principalmente a partir de un focus group dirigido a los alumnos. Entre los resultados más relevantes, destacan la superación de problemas de memoria, toma de decisiones, razonamiento y solución de problemas y metacognición, evidenciados en una gran cantidad de estudiantes, así como el mejoramiento de sus niveles de motivación en la participación en las

actividades académicas propuestas. En conclusión, la aplicación de estrategias neuropsicopedagógicas obtuvo resultados positivos en la mayoría de los alumnos que conformaron el caso de estudio.

Palabras clave: Neuropsicología, neurociencias, psicología educacional, métodos educativos, procesos cognitivos.

\section{ABSTRACT}

This research responds to the need to attend the cognitive and socio-emotional shortcomings that may be hindering the process of adaptation of newly admitted students to the university. From this identification, this study was oriented to design and apply neuropsychopedagogical strategies aimed at improving the development of students. Based on the foregoing, the general objective of this study was to describe the achievements and limitations of a proposal of neuropsychopedagogical strategies designed from an initial diagnosis and subsequently applied to university students. In this sense, the present work responds to a qualitative approach and corresponds to a research-action, which allowed to solve some problems detected in the case study, which was made up of 43 first-cycle students of the Faculty of Education of a university private of Lima. In order to detect their shortcomings, semi-structured interview guides were used for their teachers, as well as an opinion poll aimed at the students. This served as input for the design of strategies, which were evaluated mainly from a focus group aimed at the students. Among the most relevant results, we highlight the overcoming of memory problems, decision making, reasoning and problem solving, and metacognition, evidenced in a large number of students, as well as the improvement of their levels of motivation in participation in activities academic proposals. In conclusion, the application of neuropsicopedagogical strategies obtained positive results in most of the students that made up the case study.

Key Words: Neurosciences, neuropsychology, educational psychology, educational methods, cognitive processes.

\section{Introducción}

Los estudios basados en las neurociencias cognitivas aportan significativamente en el quehacer educativo al brindar conocimientos acerca del funcionamiento del cerebro y el sistema nervioso en relación con el aprendizaje. En efecto, se ha comprobado que el aprendizaje cambia la estructura cerebral; en tal sentido, mientras más aprende una persona, se vuelve más única e irrepetible (Kalbfleish, 2012; Wasserman y Zambo, 2013). Por tanto, como señalan Molina, Parra y Casanova (2017), "las barreras entre lo genético y lo ambiental se han roto" (p.121) y las condiciones del aprendizaje cobran una especial importancia. Entonces, como afirman Benarós, Lipina, Segretin, Hermida y Colombo (2010), además de Calatayud y Torres (2019), hay una necesidad de emplear los saberes propios de la neuropsicología en beneficio del aprendizaje. En tal sentido, si el docente domina estos conocimientos, podrá enfrentar mejor las situaciones 
problemáticas que observe en sus estudiantes (Paniagua, 2013; Benavidez y Flores, 2019). Desde las neurociencias, el rol del educador es el de modificador de la estructura cerebral del estudiante, organizándolo de modo adecuado para el aprendizaje. Ahora bien, en el caso de una institución de Educación Superior, se requiere una especial atención a los alumnos recién ingresantes, quienes enfrentan un proceso de adaptación a su nuevo entorno, en donde las normas y hábitos cambian radicalmente en relación con sus experiencias académicas previas. Ante ello, es preciso atender aquellos aspectos cognitivos y socioemocionales que podrían generarles algunos problemas en su proceso de acomodación a sus estudios superiores con el fin de establecer medidas orientadas a corregir o superar las limitantes que estos estudiantes podrían estar experimentando. A partir de la aplicación de las estrategias que los docentes diseñen para lograr tal objetivo, se podrá realizar una evaluación de su efectividad para, en un futuro, replicarlas en otros grupos de alumnos.

Desde un punto de vista práctico, este estudio es relevante, puesto que permite el diseño de una propuesta de estrategias neuropsicopedagógicas que docentes de cualquier asignatura de primer ciclo de nivel superior podría aplicar con sus estudiantes. De este modo, a partir del conocimiento de las deficiencias cognitivas y socioemocionales de los estudiantes -estas últimas no tomadas en cuenta en igual medida que las habilidades académicas-, se aplicó un plan de acción compuesto por estrategias diversas y articuladas, las cuales están orientadas a superar las falencias de los estudiantes.

\section{Fundamentación teórica}

Según Blakemore y Frith (2007), es importante considerar que la plasticidad del cerebro depende de cuánto se usa y no está restringida a un periodo del desarrollo. En línea con lo anterior, como recalca Salas (2003), las experiencias juegan un papel relevante en este desarrollo. De hecho, como señalan Campos (2010), Woolfolk (2010) y Paniagua (2013), las experiencias basadas en la estimulación de los sistemas sensoriales, los sistemas motores y las distintas zonas cerebrales permiten un mejor desarrollo del cerebro.

En relación con el funcionamiento cerebral durante el aprendizaje, las funciones cognitivas son aquellas que posibilitan la regulación y el control de otras habilidades y conductas. Según Pino y Urrego (2013), estos procesos facilitan la planeación, la evaluación y la modificación del comportamiento en contextos específicos, por lo cual permiten el desarrollo integral de las personas. Un primer aspecto que habría que considerar en el diseño de estrategias neuropsicopedagógicas se relaciona con dos funciones básicas: la atención y la memoria. LópezFernández y Llamas-Salguero (2018), a su vez, relacionan estas funciones cognitivas con el desarrollo de diversas competencias, como la creatividad y el lenguaje. Ahora bien, desde autores clásicos como Lakoff (1987), y Ornstein y Ehrlich (1989) hasta los más contemporáneos como Chournazidi (2016), Molina et al. (2017), y Benavidez y Flores (2019) consideran la relación directa entre atención, memoria y emociones. Así, por ejemplo, la atención puede resultar afectada por sentimientos tales como el enfado o la frustración. Tal idea está asociada con la afirmación de Ortiz (2009), quien señala que las emociones conducen a desarrollar un estado positivo o negativo hacia el aprendizaje. En efecto, ante determinados estímulos, se produce el funcionamiento de neurotransmisores que intervienen en el funcionamiento del sistema respiratorio, la temperatura, la circulación, entre otros. Ello se debe a la activación del sistema simpático, como resultado de la reacción del organismo frente a situaciones consideradas como amenazantes y que, por ende, se crea que deban ser evitadas. Tal predisposición, vinculada con las emociones y el funcionamiento de parte de su sistema neurológico, estará impidiendo el 
desarrollo de acciones necesarias para lograr un aprendizaje efectivo. En tal sentido, el componente motivacional resulta fundamental: mientras más predisposición demuestre el estudiante hacia el aprendizaje, mejor se desarrollará este proceso. Por otro lado, para almacenar información a largo plazo, habría que evaluar el nivel de significatividad de las experiencias de aprendizajes de los estudiantes (Gluck, Mercado y Myers, 2009), lo que se vincula definitivamente con las emociones involucradas en las mismas, además de sus saberes previos. "La cognición, la emoción y la conducta humana trabajan al unísono e influyen definitivamente en el desempeño del sujeto en cualquier ámbito" (Benavidez y Flores, 2019, p.28).

A partir de lo anterior, es indiscutible la necesidad de desarrollar el componente socioemocional de los estudiantes. Según Bisquerra (2003), las emociones contienen tres componentes: neurofisiológico, conductual y cognitivo. Es preciso saber que tales componentes no están separados entre sí, puesto que interactúan para reaccionar de una manera determinada ante ciertos eventos. A su vez, como menciona Salas (2003), el aprendizaje se ve influido y organizado por las emociones, además de las propias expectativas, ideologías y prejuicios. Linarez (2016) y Molina et al. (2017) señalan que el cerebro clasifica la información captada sobre la base de juicios parciales y experiencias sociales previas. Entonces, el modo en que el estudiante se enfrente al input de aprendizaje dependerá, en gran medida, de tales aspectos interiorizados. Según De la Barrera y Donolo (2009), las emociones positivas permiten intensificar las actividades de las redes neuronales y refuerzan las conexiones sinápticas. Es posible afirmar, por tanto, que el componente emotivo-valorativo es fundamental en el momento de diseñar las clases, por lo cual resulta necesario generar las condiciones óptimas para que el alumno se sienta motivado y deseoso de aprender. Si ello no ocurre, no se lograrán, al menos no de manera óptima, los aprendizajes esperados. Se sabe, asimismo, que el aprendizaje se incrementa con el desafío y se inhibe por la amenaza. Por consiguiente, es fundamental la enseñanza de estrategias de manejo de conflictos.

Los sentimientos negativos que los estudiantes manifiestan en clase no solo pueden estar relacionados con las características del profesor, sino también con las interacciones con los compañeros de clase o con la propia institución educativa. Como indican Hernández y Romero (2013), y Logatt (2016), los espacios educativos percibidos como agresivos o estresantes dificultan la concentración. Ello se debe a la presencia de "focos" que desvían la atención, lo cual convierte al entorno de aprendizaje en un lugar de olvido de todos aquellos saberes que se pretenden adquirir. En tal sentido, el aporte de las neurociencias socioafectivas resulta relevante para tal fin, pues permite reconocer todos estos signos de desatención y desmotivación de los alumnos con el fin de fomentar el desarrollo de interacciones positivas no solo profesor- alumno, sino también entre pares.

Otra habilidad cognitiva relevante es la metacognición. Esta es una destreza de alto nivel, empleada para supervisar y regular otros procesos cognoscitivos como el razonamiento, la resolución de problemas, la comprensión, entre otros. Además, implica saber qué hacer, cómo emplear la estrategia de manera oportuna y autorregular tal aplicación. Asimismo, tal como afirman Osses y Jaramillo (2008), la metacognición conduce aprender a aprender. Respecto de la metacognición, existen iniciativas interesantes orientadas a desarrollar habilidades de este tipo en estudiantes universitarios. Una de ellas es la realizada por Díaz, Pérez, González-Pienda y Núñez (2017), quienes ejecutaron un entrenamiento autorregulado con una plataforma virtual en estudiantes de primer año de tres carreras universitarias. Dicho entrenamiento consistió en desarrollar sesiones de clase orientadas a la planificación del tiempo, así como el empleo de estrategias cognitivas y metacognitivas de estudio. Los resultados fueron favorables, puesto que los investigadores demostraron que tal intervención facilitó la adquisición de destrezas de metacognición en los alumnos.

Sobre la base de todo lo anterior, en cuanto a las estrategias propuestas desde las neurociencias, habría que tomar en cuenta la clasificación realizada por Boscán (2011, como se cita en Falconi et al., 2017), las cuales se explicarán a continuación. En primer lugar, las estrategias operativas están orientadas a dotar a los estudiantes de técnicas facilitadoras de procesamiento y análisis de información, tales como organizadores gráficos, mayéutica, recursos 
mnemotéticos, entre otros. Respecto de los organizadores, resulta importante mencionar el aporte de Hernández y Romero (2013), quienes realizaron un estudio en escolares en los cuales se desarrollaron efectivamente habilidades lectoras y de escritura sobre la base del empleo de estas herramientas. En segundo lugar, las estrategias metodológicas se orientan a desarrollar habilidades de construcción del conocimiento sobre la base de recursos, tales como mapas mentales, mapas conceptuales y herramientas tecnológicas. Finalmente, las estrategias socioemocionales son aquellas que posibilitan un adecuado autodominio fisiológico, psicológico y conductual antes, durante y después de las experiencias de aprendizaje. Entre algunas de estas estrategias, destacan las técnicas de relajación, actividades de sensibilización, espacios de retroalimentación, entre otras. Por otro lado, en el diseño de estrategias, habría que considerar el estudio de Falconi et al. (2017), quienes han comprobado que la neurodidáctica es efectiva al apostar por la inversión del modo tradicional de la clase, al aprendizaje cooperativo, así como a la flexibilidad de métodos, agrupación y horarios. Esto último se relaciona con el estudio de Molina et al. (2017), quienes defienden la necesidad de respetar el tiempo de neurogénesis, el cual puede variar en cada estudiante. Es importante, por ende, favorecer el "acomodo cerebral" frente a los nuevos saberes a partir de acuerdos conjuntos con los estudiantes en cuanto a la realización de actividades de aprendizaje.

De la Caba (2001) hace referencia a un currículum de educación socioafectiva, el cual no sería posible de diseñar sin el aporte de las neurociencias socioafectivas, precisamente. Este considera, evidentemente, las etapas de desarrollo del niño y adolescente, y permite plantear un conjunto de destrezas y actitudes que deben ser fomentadas desde el aula. Un estudio que respalda la necesidad de aplicar estrategias de autorregulación emocional es el de Mateo, Canet y Laura (2015), quienes señalan que la deserción y lentificación de los estudios universitarios se deben no solo a factores cognitivos, sino también a procesos emotivos. En efecto, ellos abogan por identificar aquellas habilidades y procedimientos metacognitivos que los estudiantes manejan para, a partir de tal conocimiento, proponer un plan de acción orientado a organizar su vida académica con el fin de potenciar su rendimiento académico. Cejudo, López-Delgado, Rubio y Latorre (2015) también concuerdan con la necesidad de desarrollar la competencia socioafectiva de los estudiantes. Para ello, identificaron las percepciones de estudiantes de educación de la Universidad de Castilla-La Mancha en España sobre la importancia de la formación emocional para el logro de un adecuado desempeño docente. Entre los resultados, destacan la relevancia otorgada al desarrollo de competencias emocionales complementarias, tales como la autoestima, la gestión del estrés, la adaptabilidad a los cambios, entre otros. En segundo lugar, hallaron que los estudiantes consideraron importante la formación de habilidades interpersonales y, en un último lugar, el desarrollo de habilidades intrapersonales. Además, los alumnos encuestados consideraron la necesidad de incluir, como parte de su formación, estrategias de aprendizaje de competencias emocionales.

Resulta necesario señalar también una propuesta de intervención ejecutada por Colunga y García en 2016. El proyecto estuvo dirigido a estudiantes de postgrado de la Universidad de Camagüey en Cuba con el objetivo de favorecer sus competencias socioemocionales. A partir del diagnóstico realizado, se evidenciaron debilidades en cuanto a la autoestima formada por ellos y habilidades interpersonales, principalmente. Cabe resaltar que los talleres brindados como parte de la propuesta permitieron que los estudiantes otorguen una mayor importancia a las competencias socioafectivas y que, asimismo, se planteen metas personales para lograrlas.

Para finalizar, el estudio de Tacca, Tacca y Alva (2019) también resulta importante, ya que se orientó a conocer la relación entre las estrategias neurodidácticas con el nivel de satisfacción y rendimiento académico de estudiantes de una universidad de Lima, Perú. En los hallazgos, se pudo evidenciar que las estrategias más empleadas fueron las metodológicas; sin embargo, las que generaron mayor impacto en los estudiantes fueron las socioemocionales, lo que corrobora, una vez más, la importancia del componente emocional en el aprendizaje. 


\section{Método}

El objetivo general de este estudio fue describir los logros y limitaciones de una propuesta de estrategias neuropsicopedagógicas diseñadas a partir de un diagnóstico inicial y, posteriormente, aplicadas a estudiantes universitarios. En tal sentido, se busca responder el siguiente problema de investigación: ¿de qué manera se puede mejorar las habilidades cognitivas y socioemocionales de los estudiantes universitarios de primer ciclo de la Facultad de Educación de una universidad privada de Lima?

\section{Diseño y tipo de investigación}

El enfoque del presente estudio es cualitativo, puesto que la primera fase del estudio (diagnóstico), así como la segunda fase (aplicación de las estrategias) estuvieron orientadas a interpretar el hecho problemático en cuestión, el cual fue analizado a partir de la percepción de los participantes, así como de los docentes. Asimismo, el nivel de investigación es descriptivo, dado que se caracterizó la realidad educativa elegida, de modo que se logre un conocimiento profundo acerca de las habilidades -y carencias- cognitivas y socioafectivas que los estudiantes poseen, al igual que la efectividad de las estrategias aplicadas.

Para la etapa del diseño propiamente dicho, se consideró, como tipo de innovación, el de reforzamiento, debido a que, para mejorar las habilidades cognitivas y socioemocionales de los estudiantes, es necesario atender a la mejora de las metodologías de enseñanza de los cursos, así como consolidar el desempeño docente al respecto (Rimari Arias, 1996). Para efectos de la presente investigación, se incluyó el planteamiento de estrategias como componente importante de la metodología didáctica empleada a partir de la quinta semana de una asignatura de primer ciclo a cargo de los investigadores que asumieron el rol de docentes. De este modo, se aplicó una investigación-acción durante la aplicación de las estrategias y su consiguiente evaluación.

\section{Muestra}

Para el presente estudio, se ha considerado, como muestra a 43 estudiantes que están cursando el primer ciclo de la Facultad de Educación de una universidad privada de Lima, así como siete docentes de cursos de primer ciclo.

Es preciso resaltar que la razón por la cual se eligió trabajar con alumnos de primer ciclo es la necesidad de que estos estudiantes adquieran habilidades cognitivas y socioafectivas para desempeñarse adecuadamente a lo largo de su carrera profesional. Además, al estar estudiando la carrera de Educación, su situación es prioritaria, dado que deben superar sus propias falencias para formarse integralmente como futuros pedagogos. 
Con el propósito de recabar información acerca de las habilidades cognitivas y socioemocionales de los estudiantes desde su propia percepción, en primer lugar, se les suministró un cuestionario de ocho preguntas abiertas, orientadas a identificar lo siguiente:

- Frecuencia con que suelen distraerse en clases

- Elementos distractores más frecuentes

- Capacidad de retener instrucciones del docente

- Estrategias aplicadas para comprender los textos que lee

- Capacidad de tomar decisiones acerca de las tareas que debe ejecutar

- Habilidades de metacognición durante su proceso de aprendizaje

- Los sentimientos involucrados frente a actividades académicas

- El grado de satisfacción en su aula

En segundo lugar, se aplicó una guía de entrevista semiestructurada como instrumento de recopilación de datos provenientes de los profesores de los cursos de primer ciclo. De esta manera, se buscó identificar sus percepciones acerca del desarrollo cognitivo y socioemocional de los estudiantes, así como las estrategias didácticas aplicadas al respecto. La entrevista está compuesta de cinco preguntas abiertas, las cuales consideraron lo siguiente:

- Elementos distractores más frecuentes en los estudiantes

- Conducta de los estudiantes frente a las instrucciones proporcionadas

- Capacidad de los estudiantes de solucionar problemas

- Inclusión de estrategias neuropsicopedagógicas

Tanto el cuestionario como la entrevista fueron aplicados en la tercera semana de clases. Las categorías y subcategorías de estudio se detallan en la Tabla 1.

\section{Tabla 1}

Categorías y subcategorías de estudio

\begin{tabular}{ll}
\hline \multicolumn{1}{c}{ Categoría } & \multicolumn{1}{c}{ Subcategorías } \\
\hline Habilidades cognitivas & $\begin{array}{l}\text { Atención y memoria } \\
\text { Razonamiento y solución de problemas } \\
\text { Toma de decisiones } \\
\text { Metacognición }\end{array}$ \\
& $\begin{array}{l}\text { Tipo de motivación } \\
\text { Habilidades socioemocionales }\end{array}$ \\
& Estrategias socioemocionales \\
\hline
\end{tabular}


A partir de tal información, se diseñaron las estrategias que empezaron a ser aplicadas a partir de la quinta semana de clases. Durante la ejecución de las estrategias, se empleó un cuaderno de campo en el que se registraron las incidencias ocurridas durante las sesiones de clase, vinculadas con la aplicación de las estrategias para su próxima reacomodación según las necesidades identificadas en los estudiantes.

Finalmente, en la última semana de clases, se aplicó un focus group a la totalidad de alumnos, divididos en grupos de cinco o seis integrantes, a fin de recabar información acerca de sus percepciones respecto de la eficacia de las estrategias aplicadas. Para ello, se elaboró una guía de discusión de ocho peguntas abiertas, orientadas a evaluar el material empleado, así como las estrategias en relación con el desarrollo de habilidades de atención y memoria, razonamiento y solución de problemas, toma de decisiones, metacognición, resolución de conflictos, así como de motivación y dominio emocional. Cabe señalar que la sesión duró dos horas y media aproximadamente, y todas las respuestas fueron grabadas para su posterior análisis.

\section{Técnicas de procesamiento de información}

Luego de recabar los datos iniciales, se organizó la información. Para el análisis, se empleó el programa Atlas-Ti, el cual permitió establecer relaciones entre los datos hallados. Tal proceso facilitó la categorización e interpretación de los datos encontrados. A partir de tales insumos, se diseñaron las estrategias, las cuales fueron evaluadas, en principio, por los propios docentes investigadores a través de los registros que realizó en un cuaderno de campo. Posteriormente, tales estrategias fueron evaluadas mediante un focus group, realizado a los estudiantes implicados. Para asegurar la confiabilidad de la información hallada, tanto antes de la aplicación de las estrategias como posteriormente a esta, se aplicó una triangulación metodológica para reconocer los patrones comunes detectados en los hallazgos.

\section{Resultados}

Este apartado ha sido dividido en tres partes: descripción de las habilidades cognitivas y socioemocionales iniciales de los estudiantes sobre la base de sus percepciones manifestadas en el cuestionario, así como de las opiniones de los docentes entrevistados; el diseño de las estrategias; y la evaluación de las mismas a partir de los registros incluidos en el cuaderno de campo y principalmente del focus group.

\section{Descripción de las habilidades cognitivas y socioemocionales iniciales de los estudiantes}

Al inicio del estudio, el $41 \%$ de estudiantes manifestó poseer problemas de atención. Así, ante un estímulo externo, declararon distraerse fácilmente, lo que dificultaba su proceso de aprendizaje. Esto se reflejaba tanto en el aula, como cuando debían realizar alguna tarea en su casa, como ellos mismos indicaron. Así, les dificultaba realizar dos tareas a la vez (por ejemplo, atender la clase y escribir apuntes al mismo tiempo), además de que les costaba trabajo priorizar estímulos, 
por lo que el sonido de celular o algún ruido externo hacía que interrumpieran la atención sostenida y, asimismo, se les complicaba volver a atender la actividad que estaban realizando. Al respecto, dos de los docentes entrevistados corroboraron tal información y expresaron su incomodidad frente a este aspecto que obstaculizaba el desarrollo de las actividades académicas en las sesiones de clase. En la Figura 1, se muestra los elementos distractores principales que manifestaron los estudiantes.

En cuanto a su memoria, fue preocupante que el $51 \%$ de alumnos no recuerde ciertas indicaciones que se les comunicaba con antelación. Así, tal como expresaron, podían retener información académica en un corto tiempo, pero tal retención podía durar solo 1 o 2 días en algunos casos. Esto, a su vez, estuvo dificultando su aprendizaje, ya que, como los estudiantes mismos señalaron, varios de los aspectos que debían interiorizar para luego aplicar en la comprensión o redacción de textos, por ejemplo, se veían limitados. Al respecto, algunos docentes manifestaron su incomodidad frente al incumplimiento de tareas que ellos dejaban a los estudiantes y, en la mayoría de casos, esto se atribuyó a la falta de comprensión frente a una indicación o al olvido de las fechas planteadas.

En relación con sus habilidades de razonamiento y solución de problemas, los estudiantes mostraron dificultades también. Así, el $47 \%$ de ellos manifestaron problemas en realizar, por ejemplo, inferencias de los textos que leen. Asimismo, evidenciaron estar acostumbrados a seguir instrucciones, por lo que les cuesta trabajo comprender cómo solucionar un problema planteado sin que el docente les brinde pautas específicas. Esto último fue manifestado por dos docentes de la asignatura de Creatividad, en la cual los estudiantes no mostraban, hasta el momento de la realización del diagnóstico, la debida participación que ellos esperaban.

Figura 1.

Elementos distractores principales

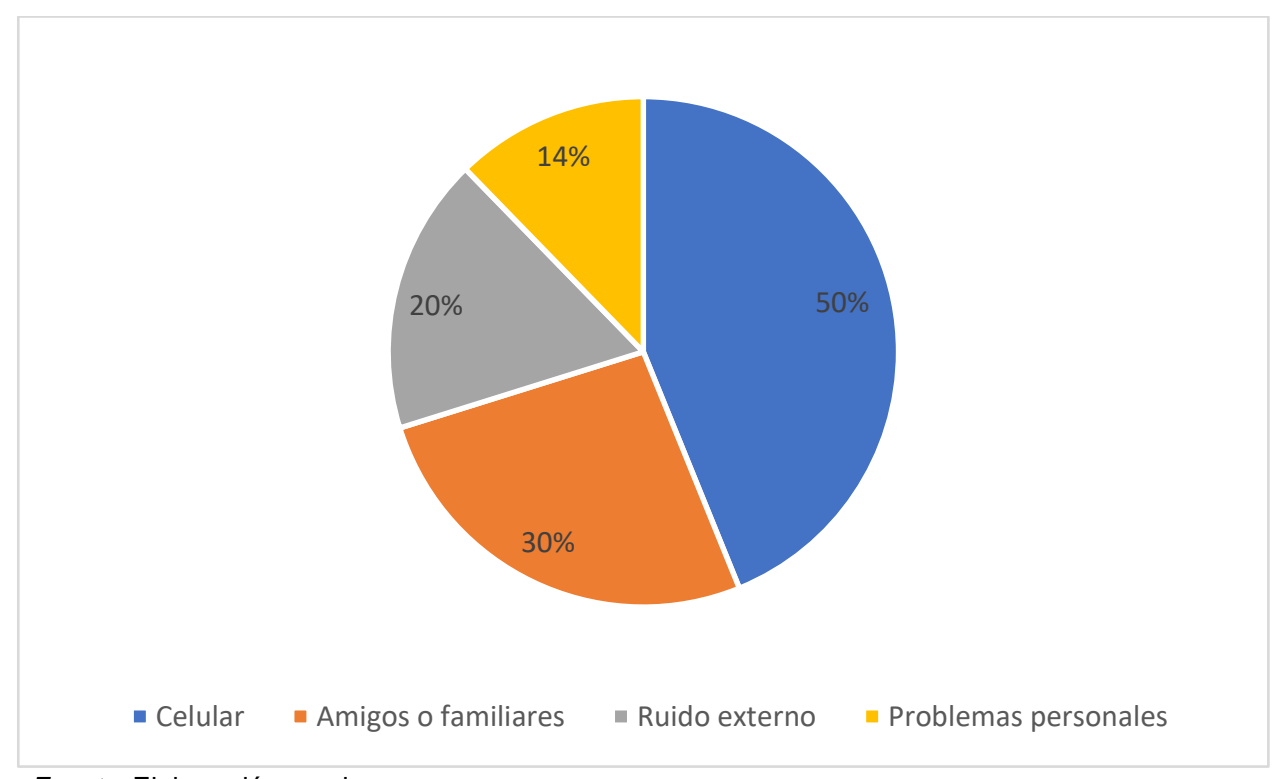

Fuente: Elaboración propia

Debido a lo planteado anteriormente, la toma de decisiones también es una función cognitiva que los alumnos aún no habían desarrollado a cabalidad. A respecto, el $35 \%$ de los estudiantes señalaron su incomodidad frente a dos de los docentes de primer ciclo, quienes, según ellos, no les daban los parámetros necesarios para realizar sus trabajos. Según señalaron, ellos preferían tener las instrucciones específicas "paso a paso" de lo que debían hacer para elaborar un trabajo académico relativo a la redacción de un ensayo. Al respecto, los profesores declararon brindar 
indicaciones generales para que los estudiantes demuestren iniciativa en la realización de los trabajos, situación que no se produce con frecuencia.

Finalmente, la metacognición es una función que aún no alcanzaban los alumnos, pues el 80 $\%$ manifestó no tener la costumbre de reflexionar acerca de su propio proceso de aprendizaje para superar las falencias de manera oportuna. Este problema está asociado con el poco espacio que se le brinda en los cursos. En efecto, 5 de los docentes entrevistados señalaron no incluir actividades propiamente metacognitivas en sus cursos, aludiendo la falta de tiempo como un factor esencial para no propiciar tales espacios.

En relación con el desarrollo socioemocional, según los docentes entrevistados, algunos alumnos presentan desmotivación, lo que genera que incumplan algunas actividades planteadas. En tal sentido, el desarrollo socioemocional de, al menos, el $40 \%$ de los estudiantes no era el adecuado. Incluso, en el cuestionario realizado, tres alumnos expresaron sufrir de ansiedad ante los exámenes, lo que los hacía comer de más o "bloquearse" totalmente, y les limitaba a enfrentar y superar situaciones problemáticas. Incluso, dos estudiantes manifestaron que les gustaría "saber cómo lograr no ponerse nerviosos" ante situaciones problemáticas que deben enfrentar día a día.

Pese a esto, los alumnos, en un $93 \%$, han señalado sentirse satisfechos con sus clases, por lo cual el clima de aula es positivo. Igualmente, las interacciones con sus pares son adecuadas: los estudiantes y docentes no han expresado casos de exclusión ni de agresión de ningún tipo. En tal sentido, las dificultades en su desarrollo socioemocional son de carácter, eminentemente, personal.

En la Figura 2, se muestra, a modo de síntesis, las dificultades presentadas por los estudiantes, lo cual requería una máxima atención.

\section{Figura 2}

Habilidades cognitivas y socioemocionales de mayor dificultad

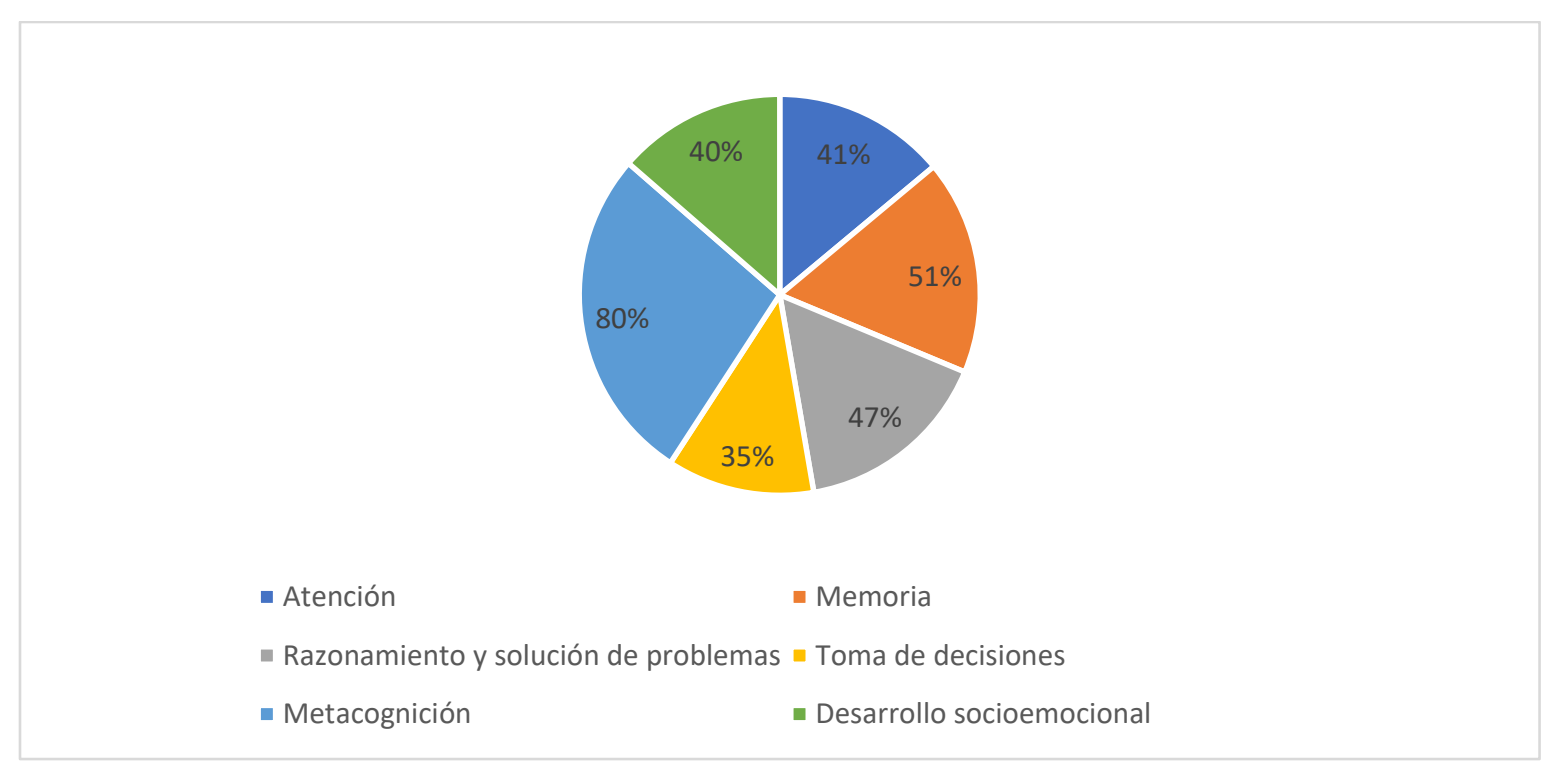

Fuente: Elaboración propia 


\section{Diseño de estrategias}

A partir de la información hallada, se diseñaron estrategias orientadas a superar las dificultades previamente mencionadas. Tales estrategias, principalmente de tipo operativo y socioemocional, estuvieron articuladas y se incorporaron en el curso de dictado de los docentes investigadores con miras a que, en un futuro, puedan ser incluidas en todas las asignaturas. Cabe señalar que el curso obedece a un aprendizaje basado en proyectos, por lo cual todas las actividades que se proponen están orientadas hacia la realización de un producto final: la elaboración de un informe de investigación acerca de una problemática de la localidad y el planteamiento de posibles soluciones. Cabe mencionar que se optó por esta metodología a fin de garantizar el desarrollo de habilidades cognitivas inherentes a este tipo de actividades, tales como toma de decisiones, resolución de conflictos, metacognición, entre otras. Igualmente, al permitir que el estudiante intervenga activamente en su propio proceso de aprendizaje, se incentiva la motivación y la responsabilidad. En la Tabla 2, se sintetiza el plan de acción en el que se incluyen algunas de las estrategias aplicadas, considerando el mes en el que se aplicó (se inició a partir del mes 2, por lo cual así está especificado en el cuadro) y el componente neuropsicológico que se estaría reforzando.

Tabla 2

Síntesis de estrategias neuropsicopedagógicas

Actividades
1. "Eligiendo mi problema de investigación"
Se solicita a los alumnos que, en equipos, conversen acerca de
los problemas de su localidad que más les preocupan. Luego,
se les pide elaborar una lista de cotejo en la que se establecen
criterios de elección del problema.
2. "Anotando la información clave"
En relación con el desarrollo de su proyecto, se pidió a los
estudiantes anotar todas las acciones que debían realizar para
lograr su producto final, junto con las fechas que ellos mismos
acordaban realizar sus entregas intermedias. Tal información
debía ser registrada en algún soporte físico o virtual.

\section{3. "Sumillando mis textos"}

Se solicita a los alumnos que, durante la lectura de textos que deben procesar en su trabajo de investigación, anoten en los márgenes sumillas para asegurarse de que están comprendiendo la información, a su vez que les servirá como estrategia para retenerla.

\section{4. "Estableciendo posibles relaciones inferenciales"}

Se solicita a los alumnos que, luego de finalizar un texto que han leído, lo relean y anoten, en los márgenes, posibles afirmaciones que se desprenden de las partes centrales del escrito. Incluso, pueden escribir posibles hipótesis que, luego, serán puestas en común en clase. Se sugiere que esta actividad, inicialmente, sea modelada por el docente.

\section{5. "Velocidad lectora"}

Con el uso de una grabadora, se realiza la lectura de un texto otorgado por alguna clase. Para ello, el estudiante deberá grabar dos párrafos leídos en voz alta teniendo en cuenta la entonación y vocalización de las palabras. La grabadora

\begin{tabular}{lllll} 
& \multicolumn{2}{c}{ Cronograma } & & Aspectos que \\
M2 & M3 & M4 & M5 & refuerzan
\end{tabular}

$X$

Toma de

decisiones

Motivación

Toma de

decisiones

Memoria

X $\mathrm{X} \quad \begin{gathered}\text { Atención y } \\ \text { memoria }\end{gathered}$

X $\quad x$

Razonamiento y solución de problemas 
(Continuación Tabla 2)

permitirá tomar el tiempo de la velocidad lectora y realizar correcciones de posibles errores, como parte de la metacognición de la lectura.

\section{6. "Detectando posibles errores de comprensión"}

Se solicita a los estudiantes que, luego de leer una fuente para su trabajo de investigación, relaten a sus compañeros de equipo aquello que han identificado como lo más importante. Luego, deberá responder las dudas. Aquello que no pueda responder será anotado por el mismo estudiante con la consigna de que, posteriormente, pueda volver a la lectura para identificar si puede responder la consulta o, en caso sea necesario, leer fuentes complementarios.

\section{7. "Evaluando mi trabajo y el de mis compañeros"}

Durante cada etapa de la realización del proyecto, se facilita a los estudiantes guías de autoevaluación y coevaluación que les permiten identificar sus propias fortalezas y debilidades (a nivel personal y de equipo), y se otorga espacios en clase para que puedan solucionar los posibles conflictos que están surgiendo.

\section{8. "Ejercicio de relajación"}

Se les pide a los estudiantes, como acción previa a la ejecución de todas sus presentaciones orales, que, en sus asientos, se pongan cómodos y peguen la espalda en el respaldar, cierren los ojos y pensar en algo positivo. Una vez ubicados en la posición adecuada, deben comenzar inhalar de manera lenta y relajada para, posteriormente, exhalar de la misma manera. Se sugiere repetir la actividad, por lo menos, unas tres veces. Asimismo, nos debemos asegurar de que la respiración sea lenta, profunda y sin ruido. Es importante incentivar este ejercicio antes de cualquier situación que pueda generarles estrés.

\section{9. "Pensamientos positivos"}

Esta actividad consiste en facilitar auto-instrucciones que permitan lograr el autocontrol y la confianza. Así, se pidió a los estudiantes repetir frases a sí mismos como "He estudiado mucho", "voy a hacerlo bien", "dejo de pensar en el bloqueo", entre otros, antes de comenzar cada presentación oral que deban realizar. Asimismo, durante la presentación, se debe recalcar que mentalmente repitan frases como las siguientes: "debo relajarme", "me siento seguro", "domino el tema" entre otros. Y, al finalizar, se sugiere recalcar a los alumnos a que lo hicieron bien, brindando prioritariamente una retroalimentación positiva (los aspectos negativos no son comunicados como tales, sino como "oportunidades de mejora").

\section{X Metacognición}

Resolución de conflictos

Toma de decisiones

Metacognición

Resolución de conflictos

$\mathrm{X} \quad$ Estrategias socioemociona les

Fuente: Elaboración propia

\section{Evaluación de las estrategias neuropsicopedagógicas}

En relación con la toma de decisiones, el $86 \%$ de los estudiantes se mostró satisfecho. Al respecto, los estudiantes manifestaron, en el focus group realizado, que las guías y fichas que elaboraron como parte de su proyecto de investigación les permitió emprender acciones pertinentes, vinculadas con la elección del problema de investigación, la búsqueda y selección de fuentes confiables, la organización de la información relevante y el producto específico que decidieron presentar. Además, la posibilidad de tomar decisiones acerca de las actividades 
intermedias que debían presentar fue percibida de modo positivo para ellos. En tal sentido, se mostraron satisfechos por el trabajo realizado durante el ciclo.

Respecto de la memoria, el $81 \%$ de los alumnos manifestó haber mejorado en recordar la información clave que los docentes de los distintos cursos les proporcionaban, gracias a las estrategias aplicadas en este proyecto. De ese porcentaje, el $65 \%$ prefirió emplear herramientas digitales en sus celulares que les permitían recordar información clave académica, así como fechas de exámenes o entregas de trabajos.

En cuanto a la atención, el $41 \%$ de estudiantes aún manifiesta tener ciertos problemas al respecto. Según ellos, no son capaces de suprimir los factores distractores que influyen en su concentración. Pese a esto, el $65 \%$ de alumnos indicó que la estrategia del sumillado les posibilitó una mejor comprensión de los textos que leyeron para efectos del proyecto de investigación. Incluso, un estudiante indicó que, a pesar de que esta técnica le exigía leer y releer el texto varias veces, ello le garantizaba una mejor comprensión de la información más importante.

En relación con sus habilidades de razonamiento y solución de problemas, el $74 \%$ de los estudiantes indicó una mejora, lo cual atribuyeron, en principio, al trabajo en equipo, lo que les permitió apoyarse mutuamente y resolver conflictos. Además, valoraron el proceso previo de reflexión que efectuaban antes de presentar cualquier tarea intermedia. Igualmente, esta habilidad se evidenció en la comprensión inferencial manifestada por los alumnos al leer sus fuentes de manera constructiva.

Con respecto a la metacognición, el 86 \% de estudiantes señaló haber comprendido cómo fortalecer esta habilidad. En efecto, al incentivarse la ejecución de actividades de reflexión constante acerca de la lectura y el aprendizaje que lograron durante el proceso de realización del proyecto, se pudo mejorar esta destreza, lo cual se vio evidenciado también en los trabajos de clase y las presentaciones orales que los estudiantes tuvieron que realizar. Pese a ello, el $51 \%$ de los alumnos indicaron que les gustaría que los demás cursos también incluyan actividades de este tipo, de modo que puedan reflexionar mejor acerca de aquello que no comprenden para proponer acciones correctivas al respecto.

En relación con la motivación, el $100 \%$ de los estudiantes manifestó satisfacción por la dinámica de trabajo realizada en el grupo. Inclusive, el $46 \%$ de los estudiantes manifestó su disconformidad con el método empleado en una o más asignaturas de primer ciclo al no propiciar debidamente un rol activo por parte de ellos.

Con respecto a la resolución de conflictos y toma de decisiones, el $86 \%$ de los estudiantes manifestó haber logrado desarrollar esta habilidad gracias a la metodología empleada. Pese a ello, 3 estudiantes manifestaron algunos problemas en el interior de su equipo que no resolvió de la manera en que ellos hubiesen preferido.

Finalmente, en cuanto a las estrategias socioemocionales, el $65 \%$ de los estudiantes manifestó haber aplicado de manera efectiva alguna de ellas. No obstante, señalaron que les gustaría conocer más estrategias para situaciones difíciles que aún les resultan estresantes, como exámenes orales o exposiciones ante una gran cantidad de personas. En la Figura 3, se muestra la mejora evidenciada por los estudiantes a partir de sus propias percepciones, considerando la evaluación inicial de diagnóstico y la evaluación final de las estrategias a partir del focus group. 


\section{Figura 3.}

Comparación entre las dificultades evidenciadas por los estudiantes después de aplicadas las estrategias y las manifestadas antes de su ejecución

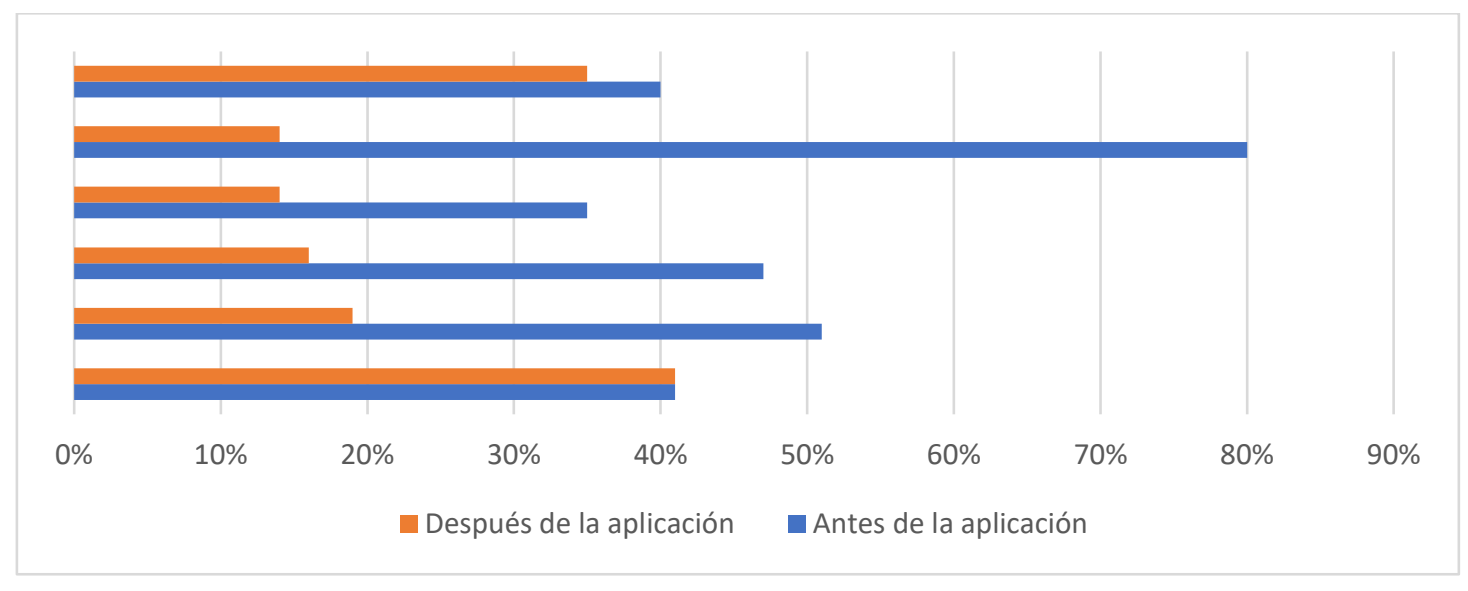

\section{Discusión}

Como afirman Salas (2003), Gluck et al. (2009), Campos (2010), Woolfolk (2010) y Paniagua (2013), las experiencias cumplen un rol fundamental en la estimulación de las distintas zonas cerebrales vinculadas con la cognición y emotividad. En tal sentido, en la medida en que se vivencien, y repitan, situaciones positivas y exitosas, se logrará una mejor disposición en estos aspectos. Por tanto, es posible afirmar que los resultados positivos de las estrategias aplicadas se deben principalmente a que las experiencias de aprendizaje favorecidas en el curso en el que estas se aplicaron resultaron satisfactorias para los estudiantes, como ellos mismos lo han manifestado. Esto se vincula directamente con el nivel de motivación positiva logrado por el $100 \%$ de los alumnos involucrados.

En principio, se identificó una mejora de la mayoría de las habilidades cognitivas, las cuales interactuaron en las distintas actividades propuestas y se logró el desarrollo de la creatividad, evidenciado en el producto final que tuvieron que presentar. Esto concuerda con el estudio de López-Fernández y Llamas-Salguero (2018), quienes sugieren la necesidad de conectar adecuadamente las funciones cognitivas para lograr la creatividad. Y es este, precisamente, uno de los objetivos del aprendizaje basado en proyectos. Ahora bien, el éxito de las estrategias también puede deberse al continuo reforzamiento positivo que se brindó a los estudiantes durante el proceso de desarrollo de su proyectos. Como se evidencia, los resultados positivos de estas habilidades se vinculan directamente con las emociones positivas manifestadas durante el proyecto, lo que concuerda con los postulados de Lakoff (1987), Ornstein y Ehrlich (1989), Chournazidi (2016), Molina et al. (2017), y Benavidez y Flores (2019). Cabe señalar que, en cuanto a la atención, las dificultades persisten; no obstante, un porcentaje considerable de los alumnos (65\%) expresó haber mejorado su atención en el momento de leer un texto, lo que se relaciona con la estrategia efectuada para este tipo de actividad.

En cuanto a la toma de decisiones, al trabajar en equipo, los estudiantes pudieron fortalecer esta habilidad, debido a que se les permitió planear, evaluar y modificar el planteamiento de las actividades que habían diseñado para la realización de su producto, lo que concuerda con Falconi et al. (2017), y Molina, et al. (2017). Según Pino y Urrego (2013), esto es muy importante, ya que replica una situación auténtica en la que los alumnos se comportan como ciudadanos potenciales REOP. Vol. 31, nº1, 1er Cuatrimestre, 2020, pp. 113 - 130 [ISSN electrónico: 1989-7448] 126 
en las que deben decidir de manera asertiva. Asimismo, ello les posibilita desarrollar otras habilidades cognitivas como el razonamiento y la solución de problemas, y la metacognición. Con respecto a esta última habilidad, el éxito evidenciado coincide con el estudio de Díaz et al. (2017), en el que se incentivó una serie de acciones autorreguladas relacionadas con la planificación del tiempo y el empleo de estrategias específicas.

En la aplicación de las estrategias diseñadas, se incentivó específicamente esta habilidades a través de estrategias autorreguladoras orientadas a que el estudiante "aprenda a aprender". No obstante, para que realmente se internalice esta habilidad y se transfiera efectivamente a diversas situaciones, es preciso el fomento de actividades orientadas a tal objetivo de manera continua, lo cual concuerda con las percepciones de los estudiantes, quienes realzaron la importancia de considerarlas en las otras asignaturas que cursan.

En cuanto al desarrollo socioemocional, las percepciones iniciales de los estudiantes concuerda con lo manifestado por Ortiz (2009), así como por De la Barrera y Donolo (2009), Mateo et al. (2015), Hernández y Romero (2013) y Logatt (2016): las emociones conducen a desarrollar un estado positivo o negativo hacia el aprendizaje. $Y$, en efecto, ante reacciones emocionales negativas frente a ciertas situaciones académicas estresantes, los alumnos señalaron su disconformidad frente a estas y a sí mismos por no poder manejarlas adecuadamente. En la aplicación de las estrategias diseñadas, pese a que la mejora en el desarrollo de destrezas socioemocionales no fue muy significativa, sí se logró un gran avance en cuanto a la motivación, pues, tal como afirma Woolfolk (2010), las experiencias satisfactorias en el ámbito académico originan percepciones positivas por parte de los estudiantes. Tal hallazgo se relaciona con el estudio de Tacca et al. (2019), quienes resaltan el grado de satisfacción que demuestran los estudiantes frente a la aplicación de estrategias neurodidácticas. Se infiere, entonces, que los estudiantes evidencian una percepción positiva acerca de la metodología de aprendizaje basado en proyectos, por lo que, si cursan alguna asignatura en la que se emplee este método, probablemente su involucramiento sea significativo y su aprendizaje, mejor. Es evidente, pues, que ellos ya formaron un juicio de valor acerca de esta dinámica de trabajo, tal como señalan Linarez (2016), y Molina et al. (2017). No obstante, es evidente la necesidad de diseñar y efectuar más actividades académicas transversales que permitan que los estudiantes refuercen su nivel de motivación y manejen de mejor manera sus emociones, sobre todo, ante las exigencias académicas que deben enfrentar.

Finalmente, como afirman Mateo et al. (2015), el truncamiento de los estudios universitarios se debe, en gran medida, a los procesos emotivos. Entonces, si los estudiantes no se sienten a gusto, probablemente no querrán continuar sus estudios. En tal sentido, el clima de clase y la metodología empleada en los cursos influyen en que los estudiantes aprendan a autorregular sus emociones. Cabe señalar que, en la presente investigación, se incentivó el logro de la autorregulación de los estudiantes precisamente considerando la relevancia del componente emocional en la percepción que los alumnos manifiesten acerca de su vida universitaria. Para ello, se plantearon actividades orientadas a ello; no obstante, como señalan Colunga y García (2016), y Cejudo et al.(2015), es preciso desarrollar un currículum de educación socioafectiva que debe ser transversal a todas las asignaturas.

\section{Conclusiones}

A partir del presente estudio, es posible reafirmar la necesidad de diseñar estrategias neuropsicopedagógicas para mejorar las habilidades cognitivas y socioemocionales de los 
estudiantes, en especial de aquellos recién ingresados en el ámbito universitario, en el cual deben ser más autónomos y responsables de su propio aprendizaje. Las estrategias diseñadas estuvieron orientadas a mejorar, por un lado, la atención, la memoria, el razonamiento y solución de problemas, la toma de decisiones y la metacognición y, por otro lado, a desarrollar la motivación, las habilidades de resolución de conflictos y la aplicación de estrategias socioemocionales para enfrentar situaciones problemáticas. A partir de los resultados, se puede concluir que es precisa la inclusión de actividades y procedimientos orientados al desarrollo de las destrezas antes mencionadas, de modo que el estudiante sea consciente de sus progresos y limitaciones. Asimismo, se ha podido comprobar que una metodología de aprendizaje basado en proyectos ayuda a mejorar muchas de estas habilidades al permitir que los alumnos asuman un rol más activo en su propio aprendizaje. No obstante, se considera que, para que realmente funcionen estas estrategias, es preciso incluirlas como parte de la práctica docente cotidiana de todos -o la mayoría- de docentes de cursos de primer ciclo. Para finalizar, cabe señalar que las estrategias neuropsicopedagógicas más efectivas fueron las orientadas a que los estudiantes lleguen a acuerdos conjuntos; analicen, paso a paso, los textos leídos; y evalúen los productos propios y de sus compañeros. El tratamiento del error, además, fue muy favorable para el desarrollo de la metacognición sobre la base de la búsqueda autónoma de estrategias orientadas a posibles fallos de comprensión o producción. En suma, es posible considerar el presente estudio como base para investigaciones posteriores en las que se propongan, de manera transversal, estrategias neuropsicopedagógicas orientadas a desarrollar habilidades cognitivas $y$ socioemocionales en estudiantes universitarios.

\section{Referencias bibliográficas}

Benarós, S., Lipina, S.J., Segretin, M.S., Hermida, M.J., y Colombo, J. A. (2010). Neurociencia y educación: hacia la construcción de puentes educativos. Revista de Neurología, 50(3), 179186.

Benavidez, V., y Flores, R. (2019). La importancia de las emociones para la neurodidáctica. Wimblu, Revista de Estudios de Psicología, 14(1), 25-53. doi: 10.15517/wl.v14i1.35935

Bisquerra, R. (2003). Educación emocional y competencias para la vida. Revista de Investigación Educativa, 21(1), 7-43. Recuperado de http://revistas.um.es/rie/article/view/99071/94661

Blakemore, S.J., y Frith, U. (2007). Cómo aprende el cerebro. Las claves para la educación. Madrid: Ariel.

Calatayud, M.A., y Torres, M.A. (2019). La neurodidáctica y la evaluación formativa. Discurso y relato de una investigación. Infancia, Educación y Aprendizaje, 5(2), 166-170. doi: 10.22370/ieya.2019.5.2.1507

Campos, A. L. (2010). Neuroeducación: uniendo las neurociencias y la educación en la búsqueda del desarrollo humano. La educación, 143, 1-14. Recuperado de http://www.educoea.org/portal/La_Educacion_Digital/laeducacion_143/articles/neuroeducacio n.pdf

Cejudo, J., López-Delgado, M.L., Rubio, M.J., y Latorre, J.M. (2015). La formación de educación emocional de los docentes: una visión de los futuros maestros. Revista Española de Orientación y Psicopedagogía, 26(3), 45-62. doi: https://doi.org/10.5944/reop.vol.26.num.3.2015.16400 
Chournazidi, A. (2016). The social framework of learning via neurodidactics. Creative Education, 7(15), 2175-2192. doi: 10.4236/ce.2016.715215

Colunga, S., y García, J. (2016). Intervención educativa para desarrollar competencias socioemocionales en la formación académica. Humanidades Médicas, 16(2), 317-335. Recuperado de http://www.medigraphic.com/cgi-bin/new/resumen.cgi?IDARTICULO=66926

De la Barrera, M.L., y Donolo, D. (2009). Neurociencias y su importancia en contextos de aprendizaje. Revista Digital Universitaria, 10(4), 1-18. Recuperado de http://www.revista.unam.mx/vol.10/num4/art20/art20.pdf

De la Caba, M.A. (2001). Intervención educativa para la prevención y el desarrollo socioafectivo en la escuela. En F. López, I. Etexebarria, M.J. Fuentes y M.J. Ortiz, Desarrollo afectivo y social (pp. 362-382). Madrid: Pirámide.

Díaz, A., Pérez, M.V., González-Pienda, J.A., y Núñez, J.C. (2017). Impacto de un entrenamiento en aprendizaje autorregulado en estudiantes universitarios. Perfiles Educativos, 39(157), 87-104. Recuperado de http://www.scielo.org.mx/scielo.php?script=sci_arttextypid=S018526982017000300087

Falconi, A., Alajo, L., Cueva, M.C., Mendoza, R.M., Ramírez, S.F., y Palma, E.N. (2017). Las neurociencias. Una visión de su aplicación en la educación. Revista Órbita Pedagógica, 4(1), 61-74. Recuperado de http://revista.isced-hbo.ed.ao/rop/index.php/ROP/article/view/89

Gluck, M.A., Mercado, E., y Myers, C.E. (2009). Aprendizaje y memoria. Del cerebro al comportamiento. México, D.F.: Mc Graw-Hill/ Interamericana Editores.

Hernández, N.A., y Romero, E. (2013). Estrategias facilitadoras del lenguaje escrito: abordaje desde la perspectiva neuropsicopedagógica. Revista Areté, 13(1), 49-60. Recuperado de https://arete.ibero.edu.co/article/view/81

Kalbfleisch, M.L. (2012). Neurotechnology in education. New York: Taylor and Francis.

Lakoff, G. (1987). Women, fire and dangerous thing. Chicago: University of Chicago Press.

Linarez, G. (2016). Aprendizaje significativo y neurociencia: la conexión del siglo XXI. Revista Iberoamericana de Producción Académica y Gestión Educativa, 3(5), 1-13.

Logatt, C.A. (2016). ¿Cómo influyen las emociones en el aprendizaje? Revista de Neurociencias y Neurosicoeducación, 83, 6-7. Recuperado de: https://www.upla.cl/inclusion/wpcontent/uploads/2016/05/Descubriendo_el_cerebro_y_la_mente_n83.pdf

López-Fernández, V., y Llamas-Salguero, F. (2018). Neuropsicología del proceso creativo. Un enfoque educativo. Revista Complutense de Educación, 29(1), 113-127. doi: http://dx.doi.org/10.5209/RCED.52103

Mateo, C., Canet, L., y Laura, M. (2015). Deserción y lentificación en los estudios universitarios: aportes cognitivos para un mejor rendimiento académico. Questión, 1(28), 147159. Recuperado de https://perio.unlp.edu.ar/ojs/index.php/question/article/view/2737

Molina, J.M., Parra, M.T., y Casanova, G. (2017). Neurodidáctica aplicada al aula en el contexto universitario. En R. Roig- Vila, J. M. Antolí, J. Blasco, A. Lledó, y N. Pellín (Eds), Redes colaborativas en torno a la docencia universitaria. (pp.115-125). Alicante: Universidad de Alicante. Recuperado de: http://hdl.handle.net/10045/70977

Ornstein, R., y Ehrlich, P. (1989). New world new mind: moving toward conscious evolution. New York: Doubleday.

Ortiz, T. (2009). Neurociencia y educación. Madrid: Alianza Editorial. 
Osses, S., y Jaramillo, S. (2008). Metacognición: un camino para aprender a aprender. Estudios Pedagógicos, 34(1), 187-197. doi: http://dx.doi.org/10.4067/S0718-07052008000100011

Paniagua, M. N. (2013). Neurodidáctica: una nueva forma de hacer educación. Fides Et Ratio, 6(6), 72-77. Recuperado de http://www.scielo.org.bo/scielo.php?pid=S2071081X2013000100009yscript=sci_abstract

Pino, M., y Urrego, Y. (2013). La importancia de las funciones ejecutivas para el desarrollo de las competencias ciudadanas en el contexto educativo. Cultura, Educación y Sociedad 4(1), 9-20. Recuperado de: https://revistascientificas.cuc.edu.co/culturaeducacionysociedad/article/view/969/pdf_189

Rimari Arias, W. (1996). La innovación educativa. Un instrumento de desarrollo. Recuperado de http://eoepsabi.educa.aragon.es/descargas/G_Recursos_orientacion/g_8_innovacion_educati va/g_8_1.docum.basicos/1.3.Innovacion_educativa_desarrollo.pdf

Salas, R. (2003). ¿La educación necesita realmente de la neurociencia? Estudios Pedagógicos, (29), 155-171. Estudios pedagógicos, (29), 155-171. doi: http://dx.doi.org/10.4067/S071807052003000100011

Tacca, D.R., Tacca, A.L., y Alva, M.A. (2019). Estrategias neurodidácticas, satisfacción y rendimiento académico en estudiantes universitarios. Cuadernos de Investigación Educativa, 10(2), 15-32. doi: http://dx.doi.org/10.18861/cied.2019.10.2.2905

Wassermann,L., y Zambo, D. (2013). Early childhood and Neuroscience. New York: Springer.

Woolfolk, A. (2010). Psicología educativa. México: Pearson Educación.

Fecha de entrada: 21 julio 2019

Fecha de revisión: 26 diciembre 2019

Fecha de aceptación: 28 diciembre 2019 